\title{
Fatal cases of human infection with avian influenza A (H7N9) virus in Shanghai, China in 2013
}

\author{
Yinzhong Shen ${ }^{1}$, Hongzhou $\mathrm{Lu}^{1, *}$, Tangkai $\mathbf{Q i}^{1}$, Yong $\mathrm{Gu}^{2}$, Ming Xiang ${ }^{2}$, Shuihua $\mathrm{Lu}^{1}$, \\ Hongping $\mathrm{Qu}^{3}$, Wenhong Zhang ${ }^{4}$, Jian $\mathrm{He}^{5}$, Huifang $\mathrm{Cao}^{6}$, Jun Ye ${ }^{7}$, Xucheng Fang ${ }^{8}$, \\ Xianzheng $\mathrm{Wu}^{9}$, Zhiyong Zhang ${ }^{1}$
}

${ }^{1}$ Shanghai Public Health Clinical Center, Fudan University, Shanghai, China;

${ }^{2}$ The Fifth Hospital of Shanghai, Fudan University, Shanghai, China;

${ }^{3}$ Ruijin Hospital, Shanghai Jiaotong University School of Medicine, Shanghai, China;

${ }^{4}$ Huashan Hospital, Fudan University, Shanghai, China;

${ }^{5}$ Changhai Hospital, Second Military Medical University, Shanghai, China;

${ }^{6}$ Jing'an District Central Hospital, Shanghai, China;

${ }^{7}$ Putuo Hospital, Shanghai University of Traditional Chinese Medicine, Shanghai, China;

${ }^{8}$ Yangpu District Shidong Hospital of Shanghai, Shanghai, China;

${ }^{9}$ Tongji Hospital, Tongji University, Shanghai, China.

Summary We retrospectively reviewed the medical records of 17 fatal H7N9 cases in Shanghai in 2013, analyzed clinical variables and described their clinical and epidemiologic characteristics. The median age was 73 years, and $82.4 \%$ had underlying medical conditions. The most frequent symptoms were fever $(100 \%)$, followed by productive cough $(\mathbf{4 7 . 1 \% )}$ and dry cough $(35.5 \%)$. Thirteen $(\mathbf{7 6 . 5} \%)$ patients had dyspnea or respiratory distress, five $\mathbf{( 2 9 . 4 \% )}$ had shock, and four $(23.5 \%)$ had acute kidney injury. Seventeen $(100.0 \%)$ patients had lymphopenia. Involvement of both lungs was found by chest radiography in $14(82.4 \%)$ patients at presentation. Fifteen $(\mathbf{8 8 . 2} \%)$ patients were hospitalized. The median times from illness onset to hospitalization and to diagnosis confirmation were both six days. Eleven (64.7\%) patients were admitted to the intensive care unit. Sixteen $(94.1 \%)$ patients were treated with oseltamivir. The median time from illness onset to oseltamivir treatment was six days. Among six patients for whom the duration of viral shedding was available, the median duration of viral shedding after oseltamivir treatment was 17 days. The median time from illness onset to death was 11 days. Refractory hypoxemia accounted for most deaths. The clinical and epidemiologic characteristics in the Shanghai fatal series of patients do not differ from other reports of $\mathrm{H} 7 \mathrm{N9}$ patients in China. This investigation reflects a delay in the diagnosis and antiviral treatment of $\mathrm{H7N9}$ patients in the early stage of the epidemic in Shanghai. Late antiviral treatment and a long duration of viral shedding may be associated with a fatal outcome in these patients.

Keywords: Avian influenza A (H7N9) virus, death, diagnosis, antiviral treatment

\section{Introduction}

The subtype H7N9 avian influenza virus has not been known to infect humans until only recently. On March

*Address correspondence to:

Dr. Hongzhou Lu, Department of Infectious Diseases, Shanghai Public Health Clinical Center, Fudan University, Shanghai 201508, China.

E-mail: luhongzhou@fudan.edu.cn
31, 2013, China confirmed the first three human cases of novel avian influenza A (H7N9) virus infection in Shanghai and Anhui, two of them have died $(1,2)$. Novel reassortant H7N9 viruses were associated with severe and fatal respiratory disease in humans, most persons with confirmed H7N9 virus infection were critically ill $(1,2)$. This increasing number of new H7N9 cases and high mortality has caused global concern and worries of spread outside of China (3). Since Shanghai reported the first case of human infection with H7N9 
virus at the end of March 2013, a total of 33 laboratoryconfirmed human cases have been reported in Shanghai in 2013 ; 18 patients $(54.5 \%)$ died while 15 were discharged from the hospital with full recovery (4). The majority of the Shanghai cases occurred between February and early April 2013 (4). To better understand the characteristics of severe influenza caused by this virus, we describe here the epidemiologic and clinical features in the fatal cases of human infection with avian influenza A (H7N9) virus in Shanghai, China in 2013.

\section{Methods}

\subsection{Ethics statement}

The study protocol was approved by the Shanghai Public Health Clinical Center Ethics Committee (SPHCCEC). Informed consent was waived by the SPHCCEC.

\subsection{Patients}

In our study, we used the case definitions of confirmed human infection with the novel H7N9 virus, which have been described by Li et al. (2). Laboratory confirmation of the novel H7N9 virus was performed with the use of the same protocols published previously $(1,2,5)$. Only patients with laboratory-confirmed infection were enrolled in this study. In 2013, 18 fatal cases of human infection with avian influenza A (H7N9) virus have been reported in Shanghai. Clinical data were available for 17 of the 18 patients. The study population included the 17 fatal cases of laboratory-confirmed influenza A (H7N9) infection diagnosed in 2013. The 17 patients received treatment in nine hospitals in Shanghai. Most patients were treated in local hospitals, but some were referred to the special hospital (Shanghai Public Health Clinical Center, Fudan University) by first medical cares after the diagnosis was confirmed.

\subsection{Data collection}

Data were collected through a review of medical records. Clinical data for confirmed cases were abstracted from original medical records with use of a data abstraction sheet. We collected information on demographic characteristics, underlying medical conditions, clinical presentation, laboratory findings, the date of illness onset, visits to clinical facilities, hospitalization, treatment and clinical outcomes.

\subsection{Laboratory evaluation}

In this study, acute kidney injury (AKI) is defined as any of the following: increase in serum creatinine (SCr) by $\geq 0.3 \mathrm{mg} / \mathrm{dL}(\geq 26.5 \mu \mathrm{mol} / \mathrm{L})$ within $48 \mathrm{~h}$; or increase in $\mathrm{SCr}$ to $\geq 1.5$ times baseline, which is known or presumed to have occurred within the prior 7 days; or urine volume $<0.5 \mathrm{~mL} / \mathrm{kg} / \mathrm{h}$ for $6 \mathrm{~h}$.

Blood cultures were obtained from all patients on admission to the hospital. Blood cultures were performed for patients presenting with chills and shivering. Sputum or endotracheal aspirates were sent for identification of possible causative bacteria or fungi.

\subsection{Statistical analysis}

SPSS software for Windows (Version 11.5; SPSS Inc., Chicago, IL) was used for statistical analysis. Continuous variables were computed with standard methods and are presented as mean and standard deviations (SD) or medians (interquartile range, IQR). For categorical variables, the percentages of patients in each category were calculated.

\section{Results}

\subsection{Epidemiologic characteristics}

The epidemiologic characteristics of the 17 patients at presentation are shown in Table 1. In Patients 3, 7, 11 and 12, the diagnosis was confirmed by means of virus isolation. All the other diagnoses were confirmed by means of nucleic acid detection. The median age of the patients was 73 years (range, 27 to 88). Most confirmed cases occurred in males $(76.5 \%)$, and $76.5 \%$ of the case patients were retirees. A total of $82.4 \%$ of the patients had one or more underlying medical conditions. Hypertension (58.8\%) and diabetes (35.3\%) were the most common underlying medical conditions. Four $(23.5 \%)$ patients reported a history of recent live poultry exposure. The time of illness onset in nine $(52.9 \%)$ patients was on or before the day when the first human infections with the novel influenza A/H7N9 virus were reported in Eastern China.

\subsection{Clinical and other features}

The most commonly reported symptoms were fever or history of fever $(100.0 \%)$, followed by productive cough $(47.1 \%)$, dry cough $(35.5 \%)$, fatigue $(17.6 \%)$ and sore throat (11.8\%) (Table 1). Muscle pain, hemoptysis, runny nose and altered consciousness were reported in one patient, respectively. No patient had diarrhea, conjunctivitis, or a rash. Physical examination revealed crackles in $13(76.5 \%)$ patients and wheezing in two (11.8\%) patients on chest examination. Thirteen (76.5\%) patients had dyspnea or respiratory distress, five (29.4\%) had shock, and four (23.5\%) had AKI. In all patients, there were marked abnormalities on chest radiography; involvement of both lungs was found by chest radiography in $14(82.4 \%)$ patients at presentation. Bilateral ground-glass opacities and consolidation were the most common radiologic findings. 


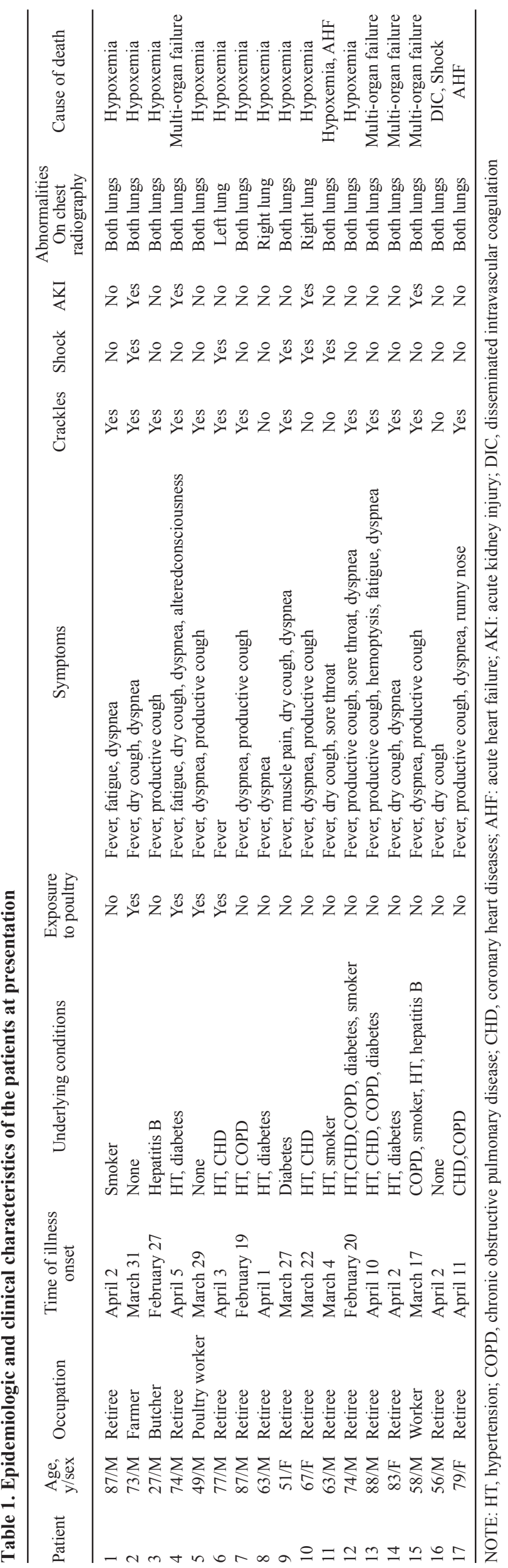

\subsection{Laboratory and microbiologic assessment}

The laboratory values of the 17 patients at presentation are shown in Table 2. Leukopenia was found in five (29.4\%) patients, $17(100.0 \%)$ had lymphopenia, two (11.8\%) had neutropenia, and eight (47.1\%) patients had thrombocytopenia. The ratios of CD4-positive cells to CD8-positive cells in Patients 2, 11, 13, 15, 16 and 17 were $8.8,0.75,2.04,2.29,3.10$ and 2.20 , respectively.

Alanine aminotransferase (ALT), aspartate aminotransferase (AST) and creatinine levels were elevated in seven $(41.2 \%), 14(82.4 \%)$ and five $(29.4 \%)$ of the 17 patients, respectively. Measurements of creatine kinase (CK) and lactate dehydrogenase (LDH) levels at presentation were available in 16 patients. CK levels were elevated in $12(75.0 \%)$ of the 16 patients. LDH levels were elevated in $16(100 \%)$ of them. C-reactive protein (CRP) levels were available in 13 patients and were elevated in $12(92.3 \%)$ of them.

Blood cultures in Patient 6 were positive for Candida albicans, all the other blood cultures were negative. Sputum cultures on admission were all negative.

\subsection{Diagnosis and treatment}

The diagnosis and treatment of the patients with avian influenza A (H7N9) are summarized in Table 3. The median time from onset of symptoms to first medical care was two days (range, 0 to 5 ). Fifteen (88.2\%) patients were hospitalized. The median time from illness onset to hospitalization was six days (range, 2 to 19$)$. Eleven $(64.7 \%)$ patients were admitted to the ICU (intensive care unit). The median time from illness onset to ICU admission was six days (range, 5 to 21). Patients 3 and 7 were the first two cases confirmed on March 31, 2013. The time from illness onset to diagnosis confirmation was 31 days in Patient 3 and 39 days in Patient 7. Patients 11 and 12 were retrospectively confirmed cases. The time from illness onset to diagnosis confirmation was 40 days in Patient 11 and 52 days in Patient 12. Among the other 13 patients, the median time from illness onset to diagnosis confirmation was six days (range, 5 to 21). Among all 17 patients, the median time from illness onset to diagnosis confirmation was six days (range, 5 to 52 ).

Sixteen $(94.1 \%)$ patients were treated with the neuraminidase (NA) inhibitor oseltamivir. The median time from illness onset to oseltamivir treatment was six days (range, 2 to 19). Only two (12.5\%) patient received oseltamivir within $48 \mathrm{~h}$ after illness onset. The median duration of oseltamivir treatment was six days (range, 1 to 30). The median dosage of oseltamivir was $150 \mathrm{mg}$ per day (range, 150 to 300 ).

The duration of viral shedding after oseltamivir treatment was 9 days, $\geq 6$ days, $\geq 13$ days, 29 days, 30 
Table 2. Laboratory values at presentation

\begin{tabular}{|c|c|c|c|c|c|c|c|c|c|c|c|c|c|c|c|c|c|}
\hline \multirow{2}{*}{ Variable } & \multicolumn{17}{|c|}{ Patient } \\
\hline & 1 & 2 & 3 & 4 & 5 & 6 & 7 & 8 & 9 & 10 & 11 & 12 & 13 & 14 & 15 & 16 & 17 \\
\hline Leukocyte (per mm³) & 5380 & 5410 & 2100 & 2700 & 2900 & 7100 & 4670 & 5620 & 3290 & 3680 & 6210 & 7110 & 7050 & 4500 & 5380 & 5480 & 6590 \\
\hline Lymphocyte (per $\mathrm{mm}^{3}$ ) & 410 & 360 & 230 & 410 & 700 & 380 & 530 & 1490 & 180 & 210 & 750 & 170 & 840 & 410 & 130 & 1140 & 260 \\
\hline Neutrophil (per $\mathrm{mm}^{3}$ ) & 4330 & 4890 & 1900 & 2620 & 2000 & 6560 & 4110 & 3430 & 3040 & 2140 & 5270 & 6810 & 5870 & 4030 & 5090 & 3730 & 6190 \\
\hline Platelet $\left(10^{3}\right.$ per $\left.\mathrm{mm}^{3}\right)$ & 186 & 71 & 58 & 192 & 71 & 102 & 78 & 94 & 155 & 162 & 390 & 67 & 166 & 121 & 75 & 137 & 79 \\
\hline Hemoglobin (g/liter) & 110 & 147 & 143 & 124 & 168 & 149 & 131 & 118 & 136 & 131 & 114 & 129 & 135 & 113 & 119 & 130 & 129 \\
\hline CK (U/liter) & 1883 & 170 & 2932 & 3889 & 1600 & 681 & 501 & 537 & 351 & 54 & 187 & 811 & 391 & NA & 772 & 290 & 170 \\
\hline LDH (U/liter) & 911 & 886 & 1683 & NA & 2150 & 640 & 480 & 433 & 525 & 444 & 620 & 651 & 505 & 1529 & 906 & 146 & 1218 \\
\hline Creatinine ( $\mu \mathrm{mol} /$ liter $)$ & 81.6 & 159.6 & 53 & 195 & 116 & 81 & 73 & 102 & 33 & 611 & 51 & 102 & 106.2 & 55 & 176.4 & 88.7 & 150.8 \\
\hline ALT (U/liter) & 27 & 20 & 71 & 103 & 76 & 132 & 31 & 54 & 80 & 33 & 34 & 33 & 41 & 54 & 35 & 25 & 37 \\
\hline AST (U/liter) & 110 & 86 & 156 & 519 & 258 & 239 & 77 & 159 & 100 & 38 & 57 & 35 & 57 & 137 & 74 & 19 & 202 \\
\hline CRP ( mg/liter) & 194 & 47 & 32 & 160 & NA & 192 & 114 & NA & NA & 4 & 134 & 220 & 196 & NA & 80.5 & 115 & 114 \\
\hline CD4:CD8 ratio & NA & 8.80 & NA & NA & NA & NA & NA & NA & NA & NA & 0.75 & NA & 2.04 & NA & 2.29 & 3.10 & 2.20 \\
\hline Myoglobulin (ng/ml) & 270 & NA & 119 & NA & 442 & 391 & NA & 229.4 & 54 & NA & NA & NA & NA & NA & 232 & 313 & 231 \\
\hline $\mathrm{BNP}(\mathrm{pg} / \mathrm{ml})$ & 1524 & NA & 158 & 301 & 871 & 423 & 7480 & NA & 327 & NA & 3000 & 10300 & NA & NA & 1309 & 194 & 7286 \\
\hline Serum amylase(U/liter) & 198 & NA & NA & 240 & 74 & NA & NA & NA & NA & NA & 224 & NA & 122 & NA & 204 & NA & 76 \\
\hline Blood culture & - & - & - & - & - & $\mathrm{CA}$ & - & - & - & - & - & - & - & - & - & - & - \\
\hline Sputum culture & - & - & - & - & - & - & - & - & - & - & - & - & - & - & - & - & - \\
\hline
\end{tabular}

NOTE: ALT, alanine aminotransferase; LDH, serum lactate dehydrogenase; AST, aspartate aminotransferase; CK, serum creatine kinase; CRP, C-reactive protein; BNP, brain natriuretic peptide; CA: Candida albicans; NA, not available; A plus sign denotes positive, and a minus sign negative.

Table 3. Diagnosis and treatment of patients infected with avian influenza A (H7N9) virus

\begin{tabular}{|c|c|c|c|c|c|c|c|c|c|c|c|c|c|c|c|c|c|}
\hline \multirow{2}{*}{ Variable } & \multicolumn{17}{|c|}{ Patient } \\
\hline & 1 & 2 & 3 & 4 & 5 & 6 & 7 & 8 & 9 & 10 & 11 & 12 & 13 & 14 & 15 & 16 & 17 \\
\hline Days from onset of symptoms to first medical care & 4 & 0 & 5 & 0 & 5 & 2 & 4 & 2 & 1 & 2 & 3 & 5 & 0 & 0 & 3 & 0 & 4 \\
\hline Hospitalization & Yes & Yes & Yes & Yes & No & Yes & Yes & No & Yes & Yes & Yes & Yes & Yes & Yes & Yes & Yes & Yes \\
\hline Days from illness onset to hospitalization & 8 & 6 & 5 & 5 & N/A & 4 & 7 & N/A & 6 & 9 & 5 & 7 & 2 & 5 & 19 & 2 & 6 \\
\hline ICU admission & Yes & Yes & Yes & Yes & No & No & Yes & No & No & Yes & No & No & Yes & Yes & Yes & Yes & Yes \\
\hline Days from illness onset to ICU admission & 8 & 6 & 7 & 5 & N/A & N/A & 8 & N/A & N/A & 9 & N/A & N/A & 6 & 5 & 21 & 6 & 6 \\
\hline Days from illness onset to diagnosis confirmation & 8 & 6 & $31^{\#}$ & 5 & 5 & 5 & $39 \#$ & 6 & 5 & 10 & $40^{*}$ & $52^{*}$ & 6 & 8 & 21 & 6 & 5 \\
\hline Oseltamivir treatment & Yes & Yes & Yes & Yes & No & Yes & Yes & Yes & Yes & Yes & Yes & Yes & Yes & Yes & Yes & Yes & Yes \\
\hline Days from illness onset to & 8 & 5 & 7 & 4 & N/A & 5 & 7 & 2 & 3 & 8 & 6 & 7 & 6 & 7 & 19 & 2 & 5 \\
\hline Durat & 9 & 5 & 4 & 2 & N/A & 6 & 6 & 4 & 1 & 11 & 2 & 4 & 13 & 7 & 29 & 30 & 21 \\
\hline Dosage of oseltamivir treatment $(\mathrm{m}$ & 150 & 150 & 150 & 300 & N/A & 300 & 150 & 150 & 150 & 300 & 150 & 300 & 300 & 150 & 150 & 150 & 150 \\
\hline Days of vir & 9 & $\geq 6$ & NA & NA & N/A & NA & NA & NA & NA & NA & NA & $\mathrm{NA}$ & $\geq 13$ & NA & 29 & 30 & 21 \\
\hline Oxygen therapy & Yes & Yes & No & Yes & N/A & No & Yes & N/A & Yes & Yes & No & Yes & Yes & Yes & Yes & Yes & Yes \\
\hline Mechanical ventilatio & Yes & Yes & No & Yes & N/A & No & Yes & N/A & Yes & Yes & No & Yes & No & Yes & Yes & No & No \\
\hline Non-invasive mechanical ventilation during follow-up & Yes & Yes & Yes & Yes & No & Yes & Yes & Yes & No & Yes & No & Yes & Yes & Yes & Yes & Yes & Yes \\
\hline Invasive mechanical ventilation during follow-up & Yes & Yes & Yes & Yes & Yes & Yes & No & No & Yes & Yes & Yes & Yes & Yes & Yes & Yes & Yes & Yes \\
\hline ECMO during & No & No & No & No & No & No & No & No & No & No & No & No & Yes & No & Yes & Yes & No \\
\hline Renal failure any time during fo & No & Yes & No & Yes & No & No & No & No & No & Yes & No & No & Yes & Yes & Yes & No & No \\
\hline Continuous renal replacement therapy & No & No & No & Yes & No & No & No & No & No & Yes & No & No & Yes & Yes & Yes & No & No \\
\hline Use of antibiotics before admission & Yes & Yes & Yes & Yes & No & Yes & Yes & Yes & Yes & Yes & Yes & Yes & Yes & Yes & Yes & No & Yes \\
\hline Use of antibiotics during hospitalization & Yes & Yes & Yes & Yes & N/A & Yes & Yes & N/A & Yes & Yes & Yes & Yes & Yes & Yes & Yes & Yes & Yes \\
\hline Use of corticosteroids any time during follow-up & Yes & Yes & Yes & No & Yes & Yes & Yes & Yes & Yes & Yes & Yes & Yes & Yes & Yes & Yes & Yes & Yes \\
\hline Intravenous immunoglobulin any time during follow-up & Yes & Yes & Yes & Yes & No & Yes & No & No & Yes & No & Yes & Yes & Yes & Yes & Yes & Yes & Yes \\
\hline Vasopressors any time during follow-up & No & Yes & No & No & Yes & Yes & Yes & No & Yes & Yes & Yes & No & Yes & No & No & No & No \\
\hline Days from onset of symptoms to death & 19 & 11 & 11 & 6 & 5 & 11 & 13 & 6 & 7 & 22 & 8 & 11 & 19 & 38 & 75 & 85 & 109 \\
\hline
\end{tabular}

NOTE: NA, not available; N/A, not applicable; ${ }^{*}$ the first two cases; ${ }^{*}$ cases confirmed retrospective; ECMO: extracorporeal membrane oxygenation.

days and 21 days in Patients 1, 2, 13, 15, 16 and 17, respectively. Influenza A (H7N9) RNA was detected in the clinical specimens using real-time reverse transcription polymerase chain reaction (rRT-PCR) 6 days and 13 days after oseltamivir treatment was intiated in Patients 2 and 13, respectively. The patients' samples were still positive for influenza H7N9 virus when the two patients died. Among the six patients for whom the duration of viral shedding was available, the median duration of viral shedding after oseltamivir treatment was 17 days.

Among the 15 hospitalized patients, 12 (80.0\%) required oxygen therapy, and nine $(60.0 \%)$ required mechanical ventilation on admission.

Fifteen $(88.2 \%)$ patients were treated empirically with broad-spectrum antibiotics both before admission and during hospitalization. Sixteen $(94.1 \%)$ patients received methylprednisolone during follow-up. Thirteen 
(76.5\%) patients received treatment with intravenous immunoglobulin, and vasopressors were used in eight (47.1\%) patients during follow-up. Patients 2, 4, 10, 13, 14 and 15 developed renal failure during follow-up, and Patients 4, 10, 13, 14 and 15 received continuous renal replacement therapy.

The median time from onset of symptoms to death was 11 days (range, 5 to 109). Refractory hypoxemia accounted for most deaths (Table 1). Neither during the period when these patients were hospitalized nor subsequently was any illness reported in a health care worker or laboratory staff member.

\section{Discussion}

This report describes 17 fatal cases of human infection with avian influenza A (H7N9) virus in Shanghai. The investigation shows that the clinical and epidemiologic characteristics in the Shanghai fatal series of patients do not differ from larger reports of H7N9 patients in China $(2,5)$. The majority of the patients were older men with preexisting medical conditions. The prominent clinical features on admission were those of a severe influenza syndrome with fever, cough, fatigue, and shortness of breath. The most striking laboratory findings were marked lymphopenia and thrombocytopenia. These clinical presentations were similar to those in the 2004 outbreak of influenza A (H5N1) in Vietnam, although diarrhea was a more prominent feature in the $\mathrm{H} 5 \mathrm{~N} 1$ patients (6). Our study shows that avian influenza A (H7N9) virus infection, characterized by multiple organ dysfunction, carries a high risk of death. The findings in this series of patients further demonstrates that novel H7N9 virus can cause severe and fatal disease in humans.

This investigation reflects a delay in the diagnosis of H7N9 patients in Shanghai. The H7N9 subtype virus has not been known to infect humans until only recently. A delayed diagnosis may be due to a weak knowledge among medical staff and the general public towards H7N9 virus associated diseases. This delay may compromise early management of patients with avian influenza A (H7N9).

This investigation also reflects a significant delay in antiviral treatment of H7N9 patients. Our previous report found that the risk of death was increased among H7N9 patients in whom antiviral therapy was initiated more than five days after illness onset (5). Our previous study also showed that the median time from the initiation of antiviral therapy to a negative test result on daily real-time RT-PCR assay was six days (5). In the current study, the median duration of viral shedding after oseltamivir treatment was 17 days. A study showed that reduction of viral load following antiviral treatment correlated with improved outcome of H7N9 patients (7). A study on timing of oseltamivir administration and outcomes in hospitalized adults with pandemic 2009 influenza A (H1N1) virus infection showed that time from onset of symptoms to oseltamivir administration was associated with a prolonged duration of fever, prolonged hospital length of stay, and higher mortality (8). A similar study showed that early oseltamivir treatment as an independent variable associated with reduced ICU mortality in critically ill patients with 2009 pandemic influenza A (9). Together with our study, these findings suggest that late antiviral treatment and a long duration of viral shedding may be associated with a fatal outcome in our case series. Therefore, early treatment of suspected or confirmed cases of avian influenza A (H7N9) is strongly recommended.

Most patients received methylprednisolone during follow-up in the current study. A study showed that emergence of NA Arg292Lys mutation in two avian influenza A (H7N9) patients who also received corticosteroid treatment led to treatment failure and a poor clinical outcome (7). The emergence of antiviral resistance in H7N9 viruses, especially in patients receiving corticosteroid therapy, is a concern. Controlled clinical studies are needed to assess the role of corticosteroids in the treatment of influenza A (H7N9) virus infections.

More aggressive treatments including oxygen therapy, mechanical ventilation, intravenous immunoglobulin, vasopressors, and continuous renal replacement therapy, have been used in patients with illness of greater severity. Most patients were treated empirically with broad-spectrum antibiotics. Antibiotic chemoprophylaxis should not be used where bacterial infection is not suspected. However, when pneumonia is present, antibiotic treatment is appropriate initially for community-acquired pneumonia.

There are some limitations to this study. First, the small case number prevents us from drawing any more conclusions. The study population is not representative of the entire H7N9 population and the results may not be generalizable. Second, the design of the study was observational, we were able to examine potential associations but were unable to assess causation. Further prospective clinical studies are needed for a better understanding of avian influenza A (H7N9) deaths.

In conclusion, the investigation shows that the clinical and epidemiologic characteristics in a Shanghai fatal series of patients do not differ from other reports of H7N9 patients in China. This investigation reflects a delay in the diagnosis and antiviral treatment of H7N9 patients in the early stage of the epidemic in Shanghai. Late antiviral treatment and a long duration of viral shedding may be associated with a fatal outcome in these patients. Strategies to facilitate rapid identification of cases and early antiviral treatment are urgently required.

\section{Acknowledgements}

This work was supported by avian influenza A 
(H7N9) grants from the Health and Family Planning Commission of Shanghai Municipality (NO: 2013QLG005).

\section{References}

1. Gao R, Cao B, Hu Y, et al. Human Infection with a novel avian-origin influenza A (H7N9) virus. N Engl J Med. 2013; 368: 1888-1897.

2. Li Q, Zhou L, Zhou M, et al. Epidemiology of human infections with avian influenza A(H7N9) virus in China. N Engl J Med. 2014, 370: 520-532.

3. Uyeki TM, Cox NJ. Global concerns regarding novel influenza A (H7N9) virus infections. New Engl J Med. 2013; 368: 1862-1864.

4. Shen Y, Lu H. Human infection with avian influenza A(H7N9) virus in Shanghai: current status and future trends. Chin Med J (Engl). 2014; 127: 1973-1976.

5. Gao HN, Lu HZ, Cao B, et al. Clinical findings in 111 cases of influenza A (H7N9) virus infection. N Engl J Med. 2013; 368: 2277-2285.

6. Tran TH, Nguyen TL, Nguyen TD, et al. Avian influenza
A (H5N1) in 10 patients in Vietnam. N Engl J Med 2004; 350: 1179-1188.

7. $\mathrm{Hu} \mathrm{Y}$, Lu S, Song Z, et al. Association between adverse clinical outcome in human disease caused by novel influenza A H7N9 virus and sustained viral shedding and emergence of antiviral resistance. Lancet. 2013; 381: 2273-2279.

8. Viasus D, Paño-Pardo JR, Pachón J, Riera M, LópezMedrano F, Payeras A, Fariñas MC, Moreno A, Rodríguez-Baño J, Oteo JA, Ortega L, Torre-Cisneros J, Segura F, Carratalà J; Novel Influenza A(H1N1) Study Group of the Spanish Network for Research in Infectious Diseases (REIPI). Timing of oseltamivir administration and outcomes in hospitalized adults with pandemic 2009 influenza A(H1N1) virus infection. Chest. 2011; 140: 1025-1032.

9. Rodríguez A, Díaz E, Martín-Loeches I, et al. Impact of early oseltamivir treatment on outcome in critically ill patients with 2009 pandemic influenza A. J Antimicrob Chemother. 2011; 66: 1140-1149.

(Received October 18, 2014; Revised January 31, 2015; Accepted February 2, 2015) 